\title{
Mode-locked Thulium Ytterbium co-Doped Fiber Laser with a Graphene Saturable Absorber
}

\author{
Ibrahim M. Babar, ${ }^{1}$ Mukul C. Paul ${ }^{* 2}$, Shyamal Das, ${ }^{2}$ Anirban Dhar, ${ }^{2}$ Harith Ahmad ${ }^{1}$ and Sulaiman W. Harun ${ }^{* 1,3}$ \\ ${ }^{1}$ Photonics Research Centre, University of Malaya,50603 Kuala Lumpur, Malaysia, \\ ${ }^{2}$ Fiber Optics and Photonics Division, CSIR-Central Glass \& Ceramic Research Institute, Kolkata, India, \\ ${ }^{3}$ Department of Electrical Engineering, University of Malaya, 50603 Kuala Lumpur, Malaysia
}

Received October 28, 2016; accepted December 30, 2016; published December 31, 2016

\begin{abstract}
A passively mode-locked Thulium Ytterbium co-doped fiber laser (TYDFL) is demonstrated using a graphene polyvinyl alcohol saturable absorber as the mode-locker. With $980 \mathrm{~nm}$ multimode pumping, the laser operates at $1942.95 \mathrm{~nm}$ with repetition rate of $11.76 \mathrm{MHz}$. The pulse width is calculated to be around $52.85 \mathrm{ps}$. The maximum pulse energy of $1190.5 \mathrm{pJ}$ is achieved at pump power of $1750 \mathrm{~mW}$.
\end{abstract}

Mode-locked fiber lasers operating at $2 \mu \mathrm{m}$ region have attracted intense interest in recent years for a number of potential applications, including atmospheric measurements, laser radar, longer-wavelength laser pumping, laser plastics, material processing, biomedical and medical applications [1-2]. Previously, a new approach was proposed to enhance Thulium-doped fiber emission at $2 \mu \mathrm{m}$ wavelength by co-doping the fiber with $\mathrm{Yb}^{3+}$ ions as a sensitizer to the $\mathrm{Tm}^{3+}$ ions [3]. This approach is feasible due to the fact that the $\mathrm{Yb}^{3+}$ emission at $1200 \mathrm{~nm}$ wavelength is one of the absorption bands of $\mathrm{Tm}^{3+}$. The excited $\mathrm{Yb}^{3+}$ ions at ${ }^{2} \mathrm{~F}_{5 / 2}$ release their energy, which is (quasi-) resonant to the $\mathrm{Tm}^{3+}$ energy level of ${ }^{3} \mathrm{H}_{5}$. In addition, the high absorption of $\mathrm{Yb}^{3+}$ ions and the uniqueness of the energy level of $\mathrm{Yb}$ provide a sufficient contribution to the high power laser application. The low quantum defect of the $\mathrm{Yb}$ enables pumping at high power.

Up to now, most ultra-short pulse fiber lasers have adopted passive mode-locking technology, in which saturable absorbers (SAs) act as a key part [4-6]. Various types of SAs have been attempted such as a semiconductor saturable absorption mirror (SESAM) [4], carbon nanotubes (CNTs) [5] and graphene [6]. Among them, SESAMs are the most widely used SA owing to their high flexibility and stability. However, SESAMs are expensive, complicated in the fabrication process and have a limited range of optical response, which limits their applications to a great extent. Developing low cost SAs for a broadband operation has always been an objective for laser experts. Recently, both single-walled carbon nanotubes (SWCNTs) and graphene have been extensively investigated for mode-locking applications

\footnotetext{
*E-mail: swharun@um.edu.my,paulmukul@hotmail.com
}

due to their various advantages, such as fast recovery time, large saturable absorption, and ease of fabrication [5-6]. Graphene is superior to CNTs in broadband saturable absorption due to its gapless linear dispersion of Dirac electrons [6]. To date, many works have been reported on the integration of a graphene polyvinyl alcohol (PVA) SA into a fiber laser system for ultra-short pulse generation. For instance, a mode-locked Erbiumdoped fiber laser with a stable soliton-like pulse was achieved using a graphene from the CVD process [7]. In this letter, a mode-locked thulium ytterbium co-doped fiber laser (TYDFL) is demonstrated by using the graphene PVA film as an SA.

The graphene is synthesized by electrochemical exfoliation of graphite at room temperature in a $1 \%$ sodium dodecyl sulfate (SDS) aqueous solution. Graphene flakes obtained from the process are mixed with PVA as the host polymer to produce free standing composite thin film which acts as a passive mode-locker in the ring laser cavity. The saturation intensity, non saturable absorption and modulation depth of the film are $971 \mathrm{MW} / \mathrm{cm}^{2}, 10 \%$ and $14 \%$, respectively. The SA is integrated in the fiber laser by sandwiching the graphene film between two fiber connectors, resulting in a stable mode-locking pulse train with a fixed repetition rate.

Figure 1 shows the experimental setup for the proposed graphene based mode-locked TYDFL. It uses a homemade double-clad thulium ytterbium co-doped fiber (TYDF) as gain medium in the ring oscillator. The fiber has an octagonal shaped pump inner cladding with a core diameter of $5.96 \mu \mathrm{m}$ and NA of 0.23 . The doping host is based on nano-engineered yttria stabilized zirconiaalumina silica glass having phase-separated nano-particles containing $\mathrm{Y}, \mathrm{Al}, \mathrm{Tm}$ and $\mathrm{Yb}$ ions rich. The selected fiber length of $10 \mathrm{~m}$ provides more than $90 \%$ pump absorption. The TYDF was pumped by a 980nm multimode laser diode through a multimode combiner (MMC). The output pulses are coupled out of the oscillator from a $20 \%$ port of the $80 / 20 \mathrm{~dB}$ output coupler while allowing $80 \%$ of the light to oscillate in the ring cavity. The length of the total cavity is set at around $17 \mathrm{~m}$ so that the net cavity dispersion is anomalous for facilitating a self-starting 
mode locked laser. For the measurements of the laser output, a fast photodetector connected to a digital oscilloscope was used to measure the pulse train and pulse waveforms. The spectrum of the output pulse was measured by an optical spectrum analyzer (OSA).

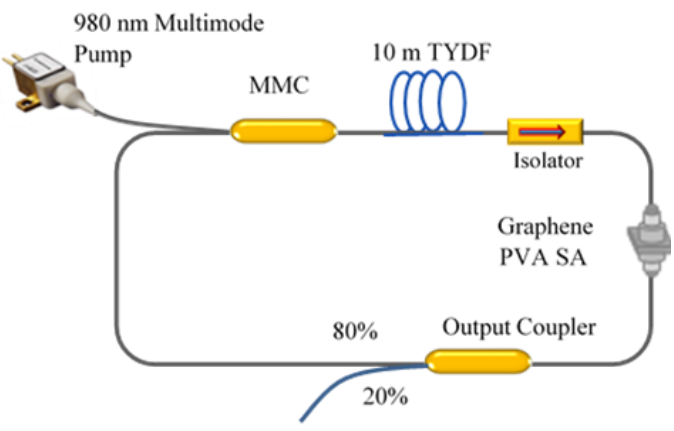

Fig. 1. The schematic setup of the mode locked TYDFL employing the fabricated graphene PVA film based SA.

Mode-locking was self-started by increasing the pump power above a threshold of $1487 \mathrm{~mW}$. The mode-locked operation was maintained as the pump power was increased up to the maximum power of $1964 \mathrm{~mW}$. Figure 2 shows the output spectrum of the mode-locked pulse train when the pump power is fixed at $1610 \mathrm{~mW}$. As shown in the figure, the spectrum was centered at $1942.95 \mathrm{~nm}$ with $3 \mathrm{~dB}$ bandwidth of $0.08 \mathrm{~nm}$. Without the $\mathrm{SA}$, the $\mathrm{CW}$ TYDFL was observed to operate at $1943.50 \mathrm{~nm}$. It is found that the operating wavelength shifts to a shorter wavelength with the insertion of the SA into the cavity due to the change in cavity loss. The oscillating laser shifts to shorter wavelength to acquire more gain to compensate for the insertion loss of the SA. With the SA, the presence of soliton is also confirmed with a weak side band at $1942.54 \mathrm{~nm}$ as shown in Fig. 2. It shows that this mode-locked fiber laser is operating in the anomalous dispersion regime. The lasing formation at a particular wavelength is depended on the TYDF gain profile and cavity loss, too. By optimizing the TYDF length and minimizing the cavity loss, the preeminent gain profile can be achieved as in the present work. A longer wavelength can be achieved if holmium is added to the TYDF.

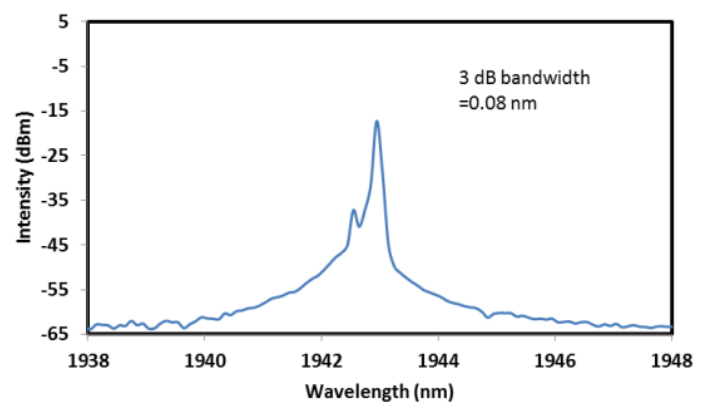

Fig. 2. Output spectrum of the mode-locked TYDFL.
The typical pulse train of the proposed graphene PVA film based passively mode-locked TYDFL is shown in Fig. 3, at a pump power of $1627 \mathrm{~mW}$. It has a pulse-topulse separation of $85 \mathrm{~ns}$, corresponding to a pulse repetition rate of $11.76 \mathrm{MHz}$, which matches the cavity roundtrip time with a cavity length of $17 \mathrm{~m}$. This indicates that the well dispersed graphene in the PVA film exhibits sufficient saturable absorption for mode-locking operation. The modulation of the pulse amplitude is an artifact caused by the limited sampling points on the sampling oscilloscope, since the pulse duration is too narrow and the sampling rate of our oscilloscope is only $1 \mathrm{Gs} / \mathrm{s}$. It was observed that the repetition rate was retained at $11.76 \mathrm{MHz}$ as the pump power was varied from 1487 to $1964 \mathrm{~mW}$. The oscilloscope trace analysis indicates that the pulse width of the laser should be less than 9ns. The actual pulse width is expected to be so much smaller than 9 ns due to the limitation of the oscilloscope resolution.

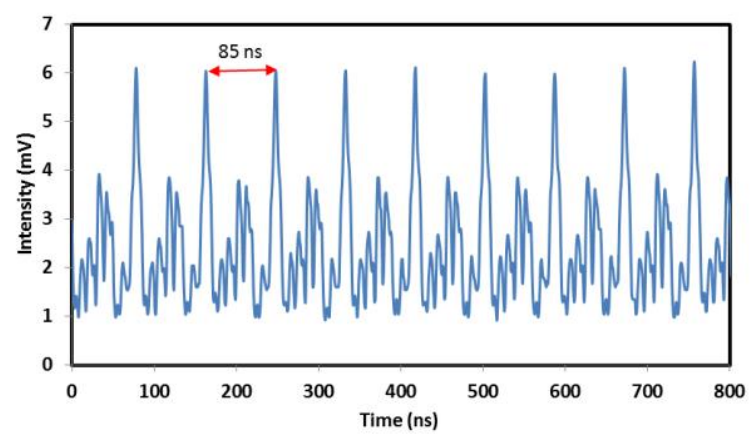

Fig. 3. Typical pulse train for the mode-locked TYDFL at pump power of $1627 \mathrm{~mW}$

The total length of the laser cavity was measured to be approximately $17 \mathrm{~m}$, comprising $10 \mathrm{~m}$ long TYDF and a $7-$ $\mathrm{m}$-long of SMF with estimated dispersions of $-0.083 \mathrm{ps}^{2} / \mathrm{m}$ and $-0.034 \mathrm{ps}^{2} / \mathrm{m}$, respectively, at $1943 \mathrm{~nm}$. Therefore, the mode-locked laser is expected to operate in an anomalous dispersion regime. On the other hand, the pulse width can be measured using an auto-correlator or can be calculated mathematically using Time Bandwidth Product (TBP). Since the Auto-correlator in $2 \mu \mathrm{m}$ range is not available, it is calculated mathematically by assuming TBP is around 0.315 for a $\operatorname{sech}^{2}$ pulse profile. The minimum pulse width possible is estimated about $51.85 \mathrm{ps}$ since the $3 \mathrm{~dB}$ bandwidth of the optical spectrum is about $0.075 \mathrm{~nm}$ $(5.96 \mathrm{GHz})$. We also calculate the repetition rate by using the formula $\mathrm{c} / 1.5 \mathrm{~L}$, found to be $11.76 \mathrm{MHz}$. This is equal to the observed repetition rate of this laser as shown in Fig. 3. The output power of the laser is also measured and the measurement is then used to calculate the single pulse energy at various pump power. The maximum output power of $14 \mathrm{~mW}$ is observed at a $1750 \mathrm{~mW}$ pump power. The pulse energy increases linearly up to a pump power of $1750 \mathrm{~mW}$ before it decreases as shown in Fig. 4. Above a 
$1750 \mathrm{~mW}$ pump power, the phase noise increases and affects the output power and pulse energy. Consequently, it degrades the pulse performance and later eliminates the pulse once the pump power reaches above $1964 \mathrm{~mW}$. The calculated pulse energy is in the range of 756.048 to $1190.476 \mathrm{pJ}$.

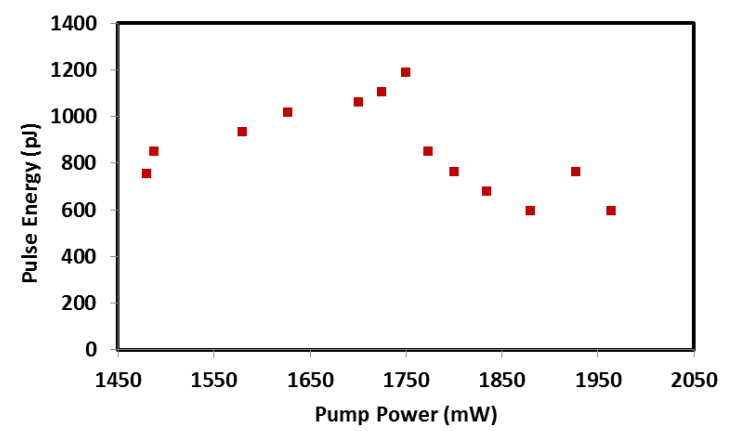

Fig. 4. The calculated pulse energy against the input pump power.

The RF spectrum of the mode-locked pulses is also measured and the result is shown in Fig. 5. Its fundamental mode peak locates at a frequency of $11.76 \mathrm{MHz}$ and has a signal-to-noise ratio (SNR) of $34 \mathrm{~dB}$, which confirms the presence of mode-locking operation in the frequency domain. Due to absorption limitation from graphene SA, the obtained SNR at $1964 \mathrm{~mW}$ is degraded. The peak of harmonic decreased moderately, which identifies the mode locked laser as having a narrow pulse width. At all pump power levels, no fundamental frequency is observed when the graphene SA is removed. Further work on reducing film loss and optimizing the TYDFL cavity is important especially for high power operation of the laser. The mode-locked pulses are generated since the longitudinal modes inside the cavity are forced to lock together. All the modes are either in phase or different in a multiple number of $2 \pi$. Even though many modes are circulating in the cavity randomly, the length of the cavity is appropriate to bring them in a relationship that can produce constructive interference between different modes. Thus no extra fiber is required to lock the circulating modes in the laser cavity.

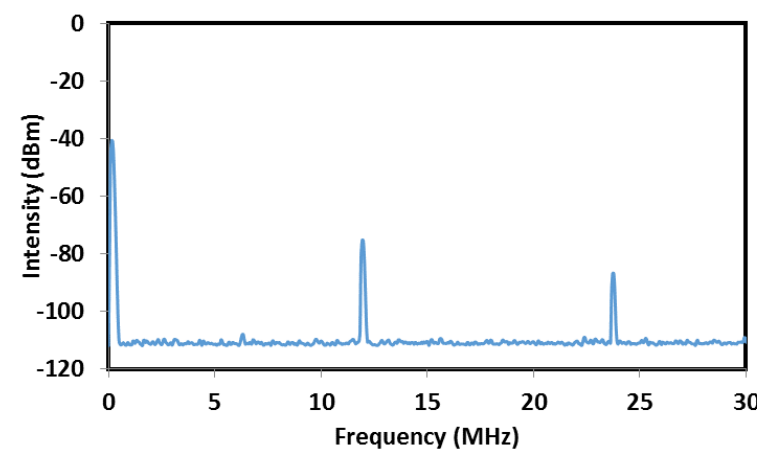

Fig. 5. RF spectrum of the mode-locked pulse train at pump power of $1964 \mathrm{~mW}$.

In conclusion, a mode-locked TYDFL operating at $2 \mu \mathrm{m}$ region is successfully demonstrated based on passive saturable absorber using an octagonal shaped double-clad nano-engineering glass based TYDF as the gain medium. The proposed laser employs a graphene PVA SA in a ring cavity with anomalous dispersion and a length of $17 \mathrm{~m}$ to generate a mode-locking pulse. The laser operates at $1942.95 \mathrm{~nm}$ with a repetition rate of $11.76 \mathrm{MHz}$ and a pulse width of around $52.85 \mathrm{ps}$. The maximum pulse energy of $1190.5 \mathrm{pJ}$ is achieved at a multimode pump power of $1750 \mathrm{~mW}$.

\section{References}

[1] J. Sotor et al., Opt. Lett. 40, 3885 (2015).

[2] J. Wang et al., Nature Scientific Reports 6, 28885 (2016).

[3] I. M. Babar et al., Ukr. J. Phys. Opt. 15, 173 (2014).

[4] Harun et al., Laser Phys. Lett. 8, 449 (2011).

[5] M. A. Ismail et al., App. Opt. 51, 8621 (2012)

[6] G. Sobon et al., Opt. Expr. 20, 19463 (2012).

[7] G. Sobon et al., Opt. Expr. 21, 12797 (2013). 